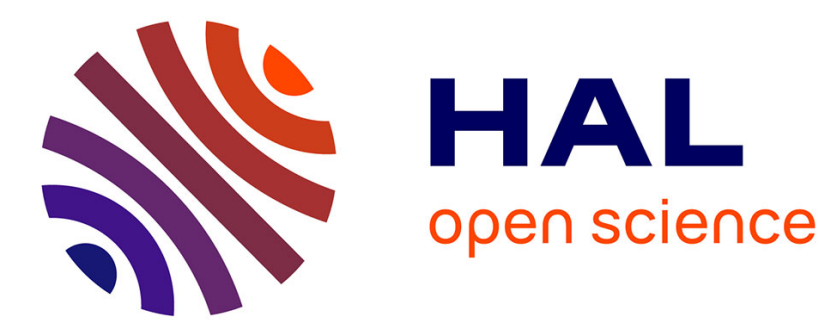

\title{
A dynamic rate adaptation algorithm using WB E-model for voice traffic over LTE network
}

Duy-Huy Nguyen, Hang Nguyen

\section{To cite this version:}

Duy-Huy Nguyen, Hang Nguyen. A dynamic rate adaptation algorithm using WB E-model for voice traffic over LTE network. 2016 Wireless Days (WD), IFIP; IEEE, Mar 2016, Toulouse, France. pp.1-6, 10.1109/WD.2016.7461490 . hal-01319420

\section{HAL Id: hal-01319420 \\ https://hal.science/hal-01319420}

Submitted on 20 May 2016

HAL is a multi-disciplinary open access archive for the deposit and dissemination of scientific research documents, whether they are published or not. The documents may come from teaching and research institutions in France or abroad, or from public or private research centers.
L'archive ouverte pluridisciplinaire HAL, est destinée au dépôt et à la diffusion de documents scientifiques de niveau recherche, publiés ou non, émanant des établissements d'enseignement et de recherche français ou étrangers, des laboratoires publics ou privés. 


\title{
A dynamic rate adaptation algorithm using WB E-model for voice traffic over LTE network
}

\author{
Duy-Huy Nguyen and Hang Nguyen \\ SAMOVAR, Télécom SudParis, CNRS, Université Paris-Saclay, 9 rue Charles Fourier - 91011 Evry Cedex \\ \{duy_huy.nguyen, hang.nguyen\}@telecom-sudparis.eu
}

\begin{abstract}
This paper presents a dynamic adaptation algorithm of joint source-channel code rate for enhancing voice transmission over LTE network. In order to assess the speech quality, we use the Wideband (WB) E-model. In this model, both end-to-end delay and packet loss are taken into account. The goal of this paper is to find out the best suboptimal solution for improving voice traffic over LTE network with some constraints on allowed maximum end-to-end delay, allowed maximum packet loss and minimum required bandwidth. The best suboptimal choice is channel code rate corresponding to each mode of the AMRWB codec that minimizes redundant bits generated by channel coding with an acceptable MOS reduction. Besides, this algorithm can be integrated with rate control in AMR-WB codec to offer the required mode of LTE network. Our results show that the MOS degradation is not significant, but the percent of reduced redundant bits to be very considerable. This will requires less bandwidth, thus, more mobile users can be served. The algorithm has simple computational operations, it can be applied to realtime voice communications.
\end{abstract}

Index Terms-AMR-WB, Wideband E-model, VoIP, VoLTE, Source-Channel code rate, Adaptive algorithm

\section{INTRODUCTION}

In present market of mobile communication in the world, 3GPP LTE (The third Generation Partnership Project Long Term Evolution) is developing strongly and is deployed by the most communications operators. LTE network is based on All-IP network and does not support circuit switching method which is utilized to provide the voice call service in $3 \mathrm{G}$ networks. So that, in order to support voice service over LTE network, additional technology has to be included. Voice over LTE network (VoLTE) service was developed to supply voice and video communication and Short Message Service (SMS) on the LTE network [1]. According to [1], there are two types of voice traffic over LTE network, those are VoLTE and VoIP. VoLTE was launched in 2012, and at present, many mobile network operators in the world have provided VoLTE service. VoLTE is VoIP (Voice over Internet Protocol) based multimedia service in which voice call and video conference services can be supplied. VoLTE is signalling protocol that enables carrying of voice packets and is guaranteed of given QoS (Quality of Service) information by LTE network operator [2]. Otherwise, VoIP relies on the internet which is done on a "best effort" basis to deliver voice packets. It is said that VoLTE is basically a subset of VoIP. It is enhanced VoIP over specific access technology (LTE) with given QoS information, but it is still VoIP.
When voice traffic is transmitted over LTE network, the voice signal firstly is compressed at Application layer by AMR-WB (Adaptive Multi-rate Wideband) codec, and then it is packetized into RTP payload. When this payload goes through each layer, it is packetized into the corresponding packet and the header is added. In order to protect the voice packet when it is delivered over a noisy channel, some error correcting technologies are included. The Forward Error Correction (FEC) channel code is widely used in LTE network for data channels is Turbo code. Channel coding reduces Bit Error Rate (BER), so that the speech quality will be improved. Channel coding encodes a $k$-bits block into a $n$-bits codeword, thus, the number of redundant bits equal to $n-k$, and so that, the code rate is $k / n$. This means that the higher channel code rate, the higher speech quality, but this leads to the longer delay and the higher redundancy. Therefore, there needs to be a tradeoff between speech quality and channel code rate.

There are several authors who have proposed techniques to improve the speech quality delivered over a noisy channel. Examples include the works of [3], [4], [5], [6], [7], and [8]. But the closest works related to our paper are represented in [3], [7], and [8]. The authors in [3] present a dynamic joint source channel coding rate adaptation algorithm for VoIP using AMR codec. The algorithm computes the optimal rates allocated to each frame for a set given QoS constraints. The aim of their paper is to find the tradeoff between packet loss recovery and end-to-end delay to maximize perceived speech quality. In [7], the authors propose an optimization issue for supplying unequal error protection of speech frames according to their importance. An optimization framework for identifying the optimal joint source-channel code rate of each voice frame based on the frame perceptual importance is proposed in [8]. In that paper, the quality of the received speech signal is maximized.

The main idea of this paper is to extend results in [3] in context of voice traffic over LTE network. Besides, instead of finding the tradeoff between packet loss recovery and endto-end delay to maximize the perceived speech quality, our proposal focuses on finding out the compromise between source code rate and channel code rate to minimize the number of redundant bits generated by channel coding with an acceptable Mean Opinion Score (MOS) degradation. It means the proposed algorithm offer a suboptimal solution for the tradeoff between speech quality and redundancy generated by channel coding. We want to offer an other point of view of 
choosing the channel code rate corresponding to each mode of AMR-WB codec for voice traffic over LTE network. In order to assess speech quality, we use the WB E-model [9]. In this model, the transmission rating factor $\left(R_{w b}\right)$ is used as a measure of subjective quality. This factor is then mapped to the corresponding MOS.

The rest of this paper is organized as follows: Overview of voice transmission over LTE network is described in section II. In section III, we present the proposed algorithm. The simulation results and performance evaluation of the proposed algorithm are analysed in section IV. The conclusion and future work is represented in section V.

\section{VOICE TRAFFIC OVER LTE NETWORK: OVERVIEW}

\section{A. Voice traffic protocol stack layers}

In LTE network, the speech frame is packetized sequentially with network protocols, including Real-time Transport Protocol (RTP), User Datagram Protocol (UDP) and Internet Protocol (IP). And then, it will be encapsulated with other radio protocols as Packet Data Convergence Protocol (PDCP), Radio Control Link (RLC) and Mac Access Control (MAC). All of these protocols will add their headers into the packetized speech packet.

In order to decrease data overhead of above protocols headers when the speech packet transmitted over a radio channel, Robust Header Compression (RoHC) is used. This will save bandwidth and enhance voice transmission over LTE network. RoHC compresses IP header from 40 bytes (with IPv4) and 60 bytes (with IPv6) down to 1 to 3 bytes [10]. For voice traffic over LTE network, HARQ (Hybrid Automatic Repeat Request) technique is used at MAC layer for retransmission if FEC fails error correction. Each speech packet will be retransmitted at least from one to three times. The retransmission times depends on the error correction or the configured maximum times of retransmission.

\section{B. Speech Codec}

Voice traffic in LTE uses AMR-WB as a vocoder. AMRWB codec is a speech codec which has been developed by ETSI (the European Telecommunications Standards Institute) and applied in the 3GPP LTE network for voice compression and decompression. It is detailed described in [11]. AMR-WB codec uses a sampling rate of $16 \mathrm{kHz}$, which covers 50-7000 $\mathrm{Hz}$ audio bandwidth. It has 9 different codec modes (from mode 0 to mode 8) corresponding to 9 source bit rates in range of $6.6-23.85 \mathrm{~Kb} / \mathrm{s}$. Each of them generates encoded 20 $\mathrm{ms}$ speech frame and switches among them every $20 \mathrm{~ms}$. The bits in the encoded speech frame to be ordered according to their subjective importance. These bits are divided into three classes with reducing perceptual importance: Class A, Class B and Class C. Total bits of each class depends on codec mode. In this study, we consider the same level of error protection for these three classes. Thus, the bits of these classes are equally protected by channel coding.

\section{LTE channel coding}

One of the crucial issues of digital communication is error correction. When data is transmitted over a noisy channel, it will be distorted by noise. Thus, the protection of data over noisy channel is mandatory. This so called is channel coding. In LTE network, encoders are used in channel coding including: Block code used for CRC (Cyclic Redundancy Code) and HARQ, Convolutional code used for control channels, with data channels, Turbo code is used. Turbo code is an enhanced Convolutional code. It is a Parallel Concatenated Convolutional Code (PCCC) with two eight-state constituent encoders and one turbo code internal interleaver, with a coding rate of $1 / 3$ [12]. Standard turbo code rates are $1 / 3,3 / 4$, and $4 / 5$, where code rate $1 / 3$ is the original code rate. Turbo code rate is chosen based on CQI (Channel Quality Indicator) index [13]. CQI index includes 16 values, where value of 0 is not used. Values of CQI index from 1 to 6,7 to 9 , and 10 to 15 are corresponding to Turbo code rates of $1 / 3,3 / 4$, and $4 / 5$. Each CQI index is mapped to a SINR (Signal-to-Interferenceplus-Noise Ratio) value. SINR also has 15 values (from -6.7 $\mathrm{dB}$ to $22.7 \mathrm{~dB}$ ) [14] counted by the receiver and sent to the transmitter. Channel coding will map from SINR value to corresponding CQI index, and then chooses the corresponding channel code rate.

In Turbo Encoder, each information bit stream is a $k$-bits block. This block size in range of 40-6144 bits. So each block can include one or several speech packets. A $k$-bits block be encoded into a $n$-bits codeword. So that, the number of redundant bits of LTE channel coding is $n-k$ bits and channel code rate is $k / n$.

\section{Wideband E-model: Speech quality assessment for Wide- band Audio}

WB E-model is a computational model developed and standardized by ITU-T [9]. It is used to estimate the MOS for wideband audio quality. The output of the model is R-factor. The values of this R-factor in range of 0-129. And then, it is mapped to the MOS. The R-factor in WB E-model is defined as follows:

$$
R_{w b}=R_{0, w b}-I_{s, w b}-I_{d, w b}-I_{e, e f f, w b}+A
$$

In which:

- $R_{0, w b}$ : The basic signal-to-noise ratio;

- $I_{s, w b}$ : The simultaneous impairment factor, it is the sum of all impairments which may occur more or less simultaneously with the voice transmission. In this model, this factor is set to 0 ;

- $I_{d, w b}$ : The delay impairment factor, representing all impairments due to delay of voice signals;

- $I_{e, e f f, w b}$ : The equipment impairment factor, capturing the effect of signal distortion due to low bit rates of the codec and packet losses of random distribution;

- $A$ : The advantage factor, capturing the fact that some users can accept a reduction of quality due to the mobility of cellular networks. In this model, this factor is set to 0 . 
In above factors, $I_{d, w b}$ and $I_{e, e f f, w b}$ are affected by endto-end delay and packet loss, respectively, while $R_{0, w b}$ and $I_{s, w b}$ do not depend on network performance. The $R_{w b}$ factor is translated into the MOS as follows [9]:

$R_{x}=R_{w b} / 1.29$

- For $R_{x}<0: M O S=1$

- For $0 \leq R_{x} \leq 100$ :

$$
\begin{aligned}
& M O S=1+0.035 \times R_{x}+7 \times 10^{-6} \times R_{x} \times \\
& \left(R_{x}-60\right) \times\left(100-R_{x}\right)
\end{aligned}
$$

- For $R_{x}>100: M O S=4.5$

$R_{w b}$ factor is mapped to the MOS using the above equation, and then, the MOS is mapped to the satisfaction level of the users. According to [15], with the wideband audio, the value of $R_{0, w b}$ factor in equation (1) equal to 129 . Thus, equation (1) can be rewritten as follows:

$$
R_{w b}=129-I_{d, w b}-I_{e, e f f, w b}
$$

In order to compute the $R_{w b}$ factor, we have to count the values of $I_{d, w b}$ and $I_{e, e f f, w b}$ factors. The $I_{d, w b}$ factor is determined by the following equation [16]:

$$
\begin{aligned}
& I_{d, w b}=0.0024 \times D_{e 2 e}+0.11 \times\left(D_{e 2 e}-177.3\right) \times \\
& H\left(D_{e 2 e}-177.3\right)
\end{aligned}
$$

In which: $H(x)$ is the Heavyside function:

$$
H(x)=\left\{\begin{array}{l}
0, \text { if } x<0 \\
1, \text { otherwise }
\end{array}\right.
$$

In equation (4), $D_{e 2 e}$ represents the total end-to-end delay (or mouth-to-ear delay) of speech packet. It will be described specifically in section III. The $I_{e, e f f, w b}$ is determined according to packet loss. In this study, we estimate packet loss probability at the receiver, after FEC. The output bits of AMRWB encoder will be encoded by channel coding (Turbo code). In fact, the bits in class A, class B and class $\mathrm{C}$ can be encoded with different channel code rates. In addition, 8-bits CRC code is applied to protect class A bits. In figure 1, the packet loss represents the average rate of speech frames for which CRC check fails in class A bits. It is determined for each mode of AMR-WB codec. According to [17], $I_{e, e f f, w b}$ is determined as follows:

$$
I_{e, e f f, w b}=I_{e, w b}+\left(129-I_{e, w b}\right) \times \frac{P_{p l}}{P_{p l}+B_{p l}}
$$

In which: $I_{e, w b}$ : The respective impairment factor without any packet loss. $P_{p l}$ : Packet loss rate. $B_{p l}$ : A codec-specific factor which characterizes its robustness against packet loss.

\section{THE PROPOSED ALGORITHM FOR MINIMIZING}

\section{REDUNDANT BITS GENERATED BY CHANNEL CODING}

As mentioned above, in the WB E-model, the value of the MOS depends on both impairment $I_{d, w b}$ and $I_{e, e f f, w b}$ factors and they have direct relationship with end-to-end delay and packet loss. If increasing end-to-end delay leads to decreasing the MOS while reducing packet loss leads to increasing the MOS. Therefore, finding out the suboptimal joint sourcechannel code rate solutions is very essential. In this paper,

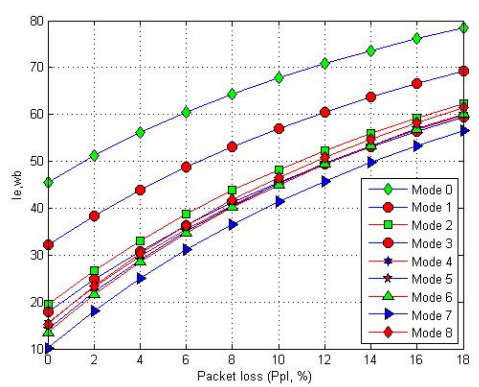

Fig. 1. $I_{e, w b}$ vs. Packet loss for nine modes of AMR-WB codec

we offer another viewpoint of choosing the suitable channel code rate corresponding to each mode of AMR-WB codec for minimizing the number of redundant bits generated by channel coding with an acceptable MOS reduction.

\section{A. The calculation of the delay impairment factor}

In order to calculate this factor, we have to compute the end-to-end delay. According to [18], the end-to-end delay can be counted as follows:

$$
D_{e 2 e}=D_{e n c}+D_{\text {network }}+D_{\text {play }}
$$

Where:

- $D_{\text {enc }}$ : The delay time caused by encoding and packetizing at the AMR-WB encoder, $D_{\text {enc }}=k \times T \times f+l_{a}+D_{\text {pack }}$ With: $T$ : The speech frame size, $T=20 \mathrm{~ms}$. $f$ : The number of frames of a speech packet. $l_{a}$ : The look-ahead delay, $l_{a}=5 \mathrm{~ms}$ for all modes of AMR-WB codec. $D_{\text {pack }}$ : The packetization delay for grouping $f$ frames into one speech packet, i.e. $D_{\text {pack }}=(f-1) \times T$.

- $D_{\text {network}}:$ The sum of transmission delay, propagation delay and queuing delay at each hop $h$ in the network path from the transmitter to the receiver. The transmission delay $\left(T_{h}\right)$ is computed using the following equation [18]: $T_{h}=(n-k+1) \times\left(T \times f \times R_{s}+H_{\text {overhead }}\right) \times \sum_{h} \frac{1}{B_{h}}$ In which: $R_{s}$ : AMR-WB bit rate before channel coding. $H_{\text {overhead }}$ : The number of overhead bits introduced by RTP/UDP/IPv6/PDCP/RLC/MAC headers and 24 bits introduced by $\mathrm{CRC}$ at Physical layer. $B_{h}$ : The bandwidth at hop $h$.

The propagation delay $\left(P_{h}\right)$ depends on the distance from the source to destination. It is negligible if within a local area. For intra-continental calls, the propagation delay is in the order of $30 \mathrm{~ms}$ and for inter-continental calls, it can be as large as $100 \mathrm{~ms}$ [18]. It is clear that for a given voice connection, the only random component of voice delay (that is the only source of jitter) consists of queuing delay in the network [18], $Q=\sum_{h} Q_{h}$.

- $D_{\text {play: }}$ The playback delay, voice packets are usually delayed in a jitter buffer and the fixed playback delay must be equal to at least two speech frame length [19], i.e. $D_{\text {play }}=2 \times T$. 
From above analysis, we get the end-to-end delay determined as the following equation:

$$
\begin{aligned}
& D_{e 2 e}=k \times T \times f+l_{a}+(n-k+1) \times\left(T \times f \times R_{s}+\right. \\
& \left.H_{\text {overhead }}\right) \times \sum_{h} \frac{1}{B_{h}}+\sum_{h}\left(Q_{h}+P_{h}\right)+2 \times T+D_{\text {rohc }}+ \\
& D_{\text {harq }}
\end{aligned}
$$

With $D_{\text {rohc }}$ : The delay of RoHC processing time at PDCP layer. According to [20], RoHC should not noticeably add to the end-to-end delay and according to [21], this delay is not very significant, approximately $10-67 \mu \mathrm{s} /$ packet to compress and $12-51 \mu \mathrm{s} /$ packet to decompress RoHC packets. Thus, in this study, we consider the delay caused by RoHC equal to $0 . D_{\text {harq }}$ : The delay due to retransmission at MAC layer by HARQ. Each voice packet is retransmitted at least one times. According to [22], because the RTT (round trip time) of HARQ is fixed and because of the higher priority for retransmissions, the HARQ delay is normally within $10 \mathrm{~ms}$.

Normally, FEC also can cause the delay. However, a number of authors have pointed out that FEC does not introduce any delay unless there is packet loss. So that, in this study, we do not mention the delay caused by FEC. After calculating the end-to-end delay, we can obtain the impairment factor $I_{d, w b}$ from equation (4).

\section{B. The calculation of the equipment impairment factor}

In order to compute this factor, we have to count the packet loss rate after FEC schemes try to recover errors. We assume that the estimates for the packet loss rate $P_{p l}$ on the end-toend network path is available at time an adaptation decision is being made. In order count the $P_{p l}$, we assume a random loss model, the relationship between the parameters $(k, n)$ of FEC schemes, "raw" packet loss rate on the end-to-end network path $p_{r}$ and the packet loss rate $P_{p l}$ is described as follows [20]:

$$
P_{p l}=\sum_{i=n-k+1}^{n}\left(\begin{array}{l}
n \\
i
\end{array}\right) \times p_{r}^{i} \times\left(1-p_{r}\right)^{n-i} \times \frac{i}{n}
$$

Equation (9) shows that, we can attain the current $p_{r}$ from the given measurement $P_{p l}$ and a pair of $(k, n)$. This $p_{r}$ value is counted once per adaptation period and is utilized in the proposed algorithm as shown in the next subsection. Figure 1 shows $I_{e, w b}$ versus packet loss(\%) after FEC. Thus, we can obtain $I_{e, w b}$ by referring to figure 1 .

\section{The proposed algorithm}

In this algorithm, we solve the problem of choosing suitable source and channel code rate within some constraints on maximum allowed end-to-end delay, maximum permitted packet loss and minimum required bandwidth for minimizing the number of redundant bits generated by channel coding with an acceptable MOS reduction. This algorithm is located at the transmitting side. The inputs of the algorithm include:

- QoS information: As path packet loss $P_{p l}$, path bandwidth $B_{h}$ and congestion $Q_{h}$. These values can obtain by QoS estimation module used in the network.
- QoS constraints: Maximum allowed end-to-end delay $D_{\max }$, maximum permitted packet loss rate $P_{\max }$ and minimum required bandwidth $B W_{r}$.

The outputs of the algorithm will be a decision on the choice of source code rate $R_{s}$ and channel code rate $R_{c}$. Besides the most suboptimal choice of channel code rate for every AMRWB codec mode, the algorithm also offers the best suboptimal choice of channel code rate for all of AMR-WB codec modes. In order to describe the algorithm, we start by setting two the following constraints:

$$
\left\{\begin{array}{l}
D_{e 2 e} \leq D_{\max } \\
P_{p l} \leq P_{\max }
\end{array}\right.
$$

For each mode in AMR-WB codec modes, we change $k$ and $n$ over ranges $k=1,2, \ldots, k_{0}$ and $n=k+1, \ldots, n_{0}$ where $k_{0}$ and $n_{0}$ are maximum available values of channel code rate in LTE network $\left(n_{0}>k_{0}\right)$. In order to find the most suitable suboptimal pair of $\left(k_{\text {subopt }}, n_{\text {subopt }}\right)$ for all values of $R_{s}$, we define the MOS reduction $\left(M O S_{r}, \%\right)$ and the percent of decreased redundant bits $\left(G_{r}, \%\right)$ as follows:

$$
\begin{gathered}
M O S_{r}=\frac{\operatorname{MOS}\left(k_{\text {best }}, n_{\text {best }}\right)-\operatorname{MOS}\left(k_{\text {subopt }}, n_{\text {subopt }}\right)}{M O S\left(k_{\text {best }}, n_{\text {best }}\right)} \times 100 \\
G_{r}=\frac{\left(n_{\text {best }}-k_{\text {best }}\right)-\left(n_{\text {subopt }}-k_{\text {subopt }}\right)}{n_{\text {best }}-k_{\text {best }}} \times 100
\end{gathered}
$$

We see that if the larger $M O S_{r}$, the lower speech quality, and the higher reduced redundant bits, the higher BER (Bit Error Rate) and this leads to the lower speech quality. So that, in order to find out the tradeoff between $M O S_{r}$ and $G_{r}$, we propose the criteria for this as follows: In the pairs of $\left(k_{\text {subopt }}, n_{\text {subopt }}\right)$ of all modes, the algorithm firstly finds a pair which has the maximum value of $G_{r}$ meeting $M O S_{r} \leq$ $1 \%$ to ensure that this MOS is very close to the highest MOS. In the case there are many the same maximum values of $G_{r}$, the algorithm will secondly choose a pair of $\left(k_{\text {subopt }}, n_{\text {subopt }}\right)$ which has the lowest value of the $M O S_{r}$. If there are many the same maximum values of $M O S_{r}$, then the algorithm will thirdly choose a pair of $\left(k_{\text {subopt }}, n_{\text {subopt }}\right)$ which has the lowest value of $B W_{\text {subopt }}$ where $B W_{\text {subopt }}$ is the required bandwidth corresponding to the pair of $\left(k_{\text {subopt }}, n_{\text {subopt }}\right)$. The required bandwidth of each mobile user $\left(B W_{r}\right)$ must be met the following condition:

$$
B W_{r} \geq \frac{n}{k} \times\left(R_{s}+\frac{H_{\text {overhead }}}{T \times f}\right)
$$

In which: all factors are as same as ones described in the previous equations.

Finally, the proposed will offer the best suboptimal solution for minimizing the redundant bits generated by channel coding with an acceptable MOS reduction. The steps of the proposed algorithm are described in Algorithm 1.

\section{Simulation Results and Performance EVALUATION}

In order to simulate the algorithm, we assume simulation parameters as follows:

- The number of speech frames per packet: $f=1$ 

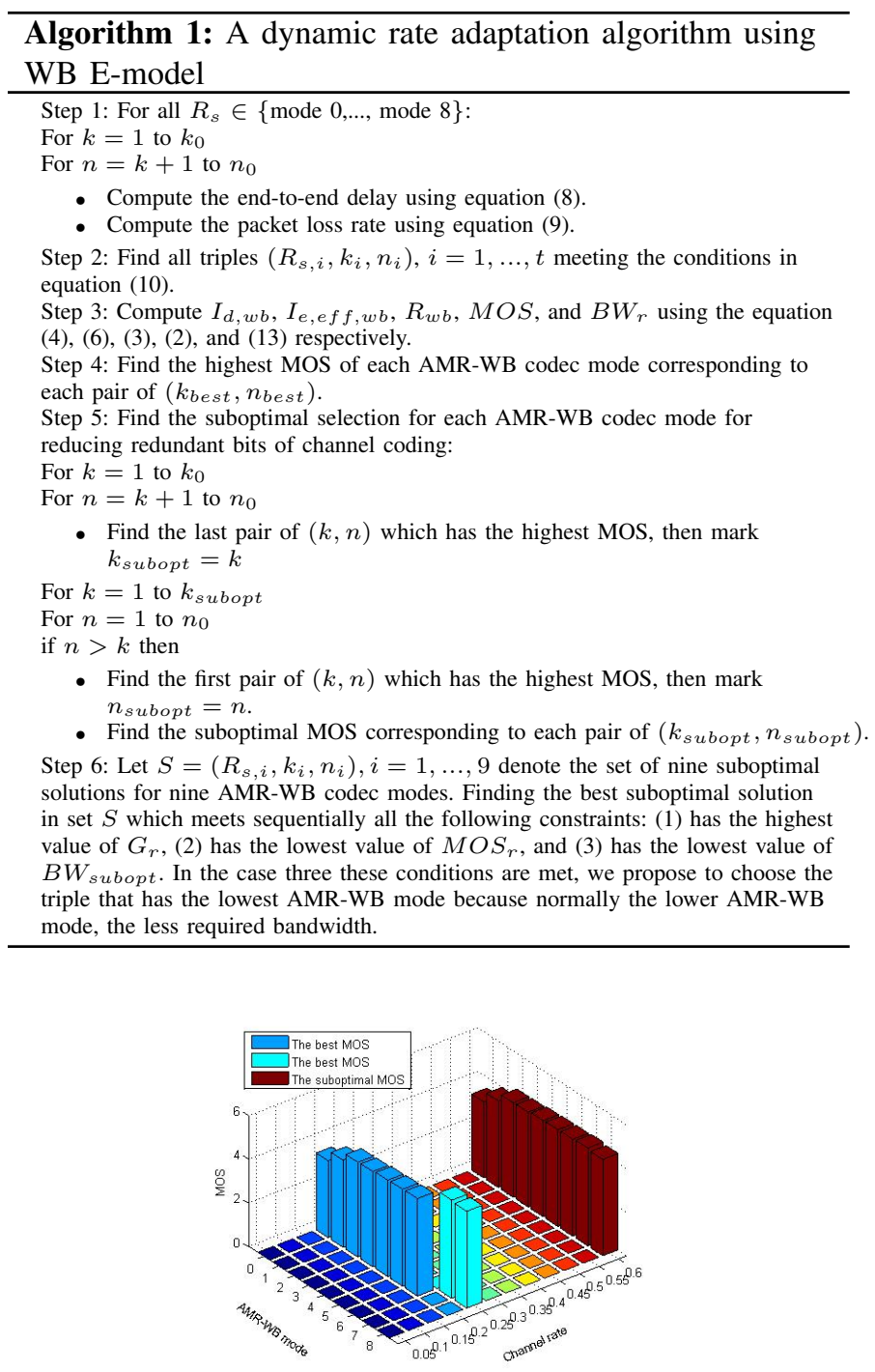

Fig. 2. MOS vs. AMR-WB mode and channel code rate when $p_{r}$ is fixed equal to $10 \%$

TABLE I

THE DETAILED RESULTS OF FIGURE 2

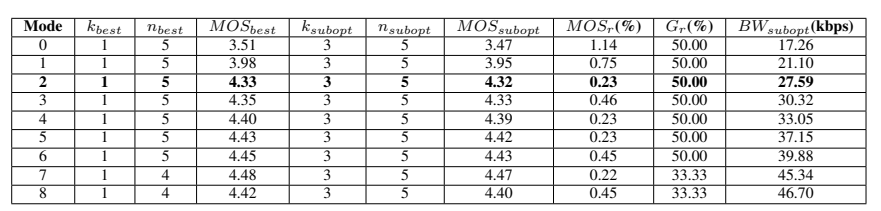

- The network path includes 15 hops, with 13 hops are the fast core network links: 10 hops at $622 \mathrm{Mb} / \mathrm{s}$, and 3 hops at $1.8 \mathrm{~Gb} / \mathrm{s}$, and two are in the eNodeB cell at $50.4 \mathrm{Mb} / \mathrm{s}$ and $25 \mathrm{Mb} / \mathrm{s}$, respectively.

- $P_{h}=0.06 \mathrm{~ms}$ per hop. $Q_{h}$ is random between 0 and 1 $\mathrm{ms}$.

- $D_{\max }=150 \mathrm{~ms} . P_{\max }=10^{-2}$

- $k_{0}=4$ and $n_{0}=5$

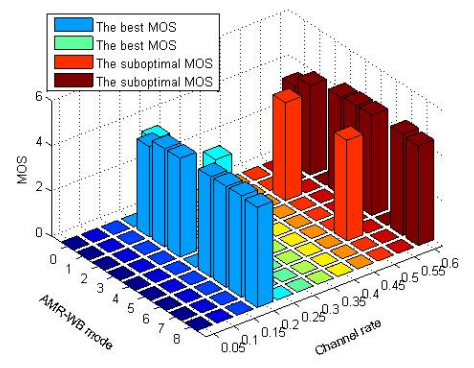

Fig. 3. MOS vs. AMR-WB mode and channel code rate when $p_{r}$ is randomly changed in range of $0 . .15 \%$
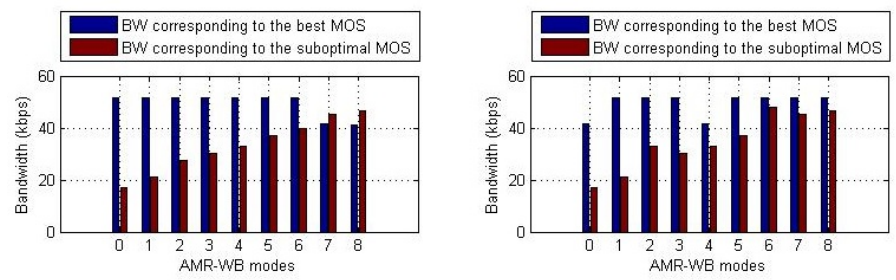

Fig. 4. AMR-WB mode vs required bandwidth when $p_{r}$ is fixed equal to $10 \%$ (left figure) and when $p_{r}$ is randomly changed in range of $0 . .15 \%$ (right figure)

TABLE II

THE DETAILED RESULTS OF FIGURE 3

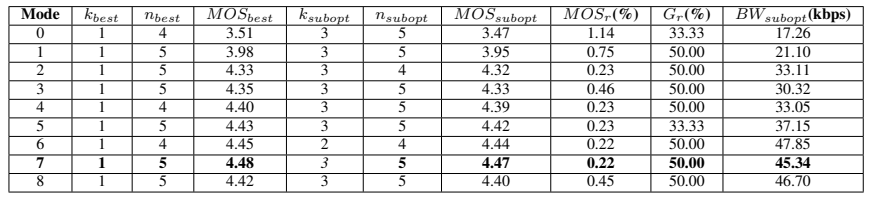

Figure 2 and table I show the results in the case "raw" packet loss is fixed equal to $10 \%$. This demonstrates that when the "raw" packet loss is fixed, the best MOS depends on only two channel code rates, and one for the suboptimal MOS. Figure 2 also shows that with the "raw" packet loss is fixed equal to $10 \%$, all of AMR-WB codec modes obtain the best MOS with channel code rate equal to $1 / 5$ and $1 / 4$, and the suboptimal MOS with channel code rate equal to $3 / 5$. The results show that the suboptimal MOS is very close to the best MOS while the redundant bits generated by channel coding decreased significantly. In this case, the $G_{r}$ of all triples have two different values $(50 \%$ and $33.33 \%)$. There are only modes of 1 to 6 that have the highest values of $G_{r}$, thus, the algorithm will choose according to the lowest values of $M O S_{r}$. There are modes of 2, 4, and 5 that have the lowest values of $M O S_{r}(0.23 \%)$, the algorithm will choose according to the third condition that has the lowest value of $B W_{\text {subopt }}$. So that, the triple $\left(R_{s, 2}, 3,5\right)$ is the best suboptimal solution because it has the lowest value of $M O S_{r}$ with the suboptimal MOS equal to 4.32 while the best MOS equal to 4.33. Figure 3 and table II show the results in the case the "raw" packet loss is varied in an instant. These results demonstrate that when the "raw" packet loss is changed, the best MOS depends on 
only two specific channel code rates $(1 / 4$ and $1 / 5)$, while the suboptimal MOS depends on three channel code rates $(3 / 5,3 / 4$, and $2 / 4)$. The results also show that there are 7 triples which have the same highest value of $G_{r}(50 \%)$, those are two triples of $\left(R_{s, 1}, 3,5\right),\left(R_{s, 2}, 3,4\right),\left(R_{s, 3}, 3,5\right)$, $\left(R_{s, 4}, 3,5\right),\left(R_{s, 6}, 2,4\right),\left(R_{s, 7}, 3,5\right)$ and $\left(R_{s, 8}, 3,5\right)$ which meet the conditions. Similar to the case of the fixed pr, the algorithm will firstly choose triples that have the highest values of $G_{r}$, secondly the lowest values of $M O S_{r}$ and thirdly the lowest values of $B W_{\text {subopt }}$. So that, in this case, the best suboptimal solution is triple of $\left(R_{s, 7}, 3,5\right)$ which has the highest value of $G_{r}(50 \%)$, the lowest values of $M O S_{r}$ (0.22\%), and the lowest values of $B W_{\text {subopt }}$ (45.34) with the suboptimal MOS equal to 4.47 while the best MOS equal to 4.48 .

In order to demonstrate that the proposed algorithm saving the bandwidth, we calculate the minimum required bandwidth for two cases of the best MOS and the suboptimal MOS. These values can be obtained from the equation (13) through two couples of $\left(k_{\text {best }}, n_{\text {best }}\right)$ and $\left(k_{\text {subopt }}, n_{\text {subopt }}\right)$. Figure 4 shows the charts of the minimum required bandwidth of each mode of AMR-WB codec corresponding to two cases of the best MOS and the suboptimal MOS. It is clear that, when we choose according to the suboptimal condition, the system will save the bandwidth very significantly. This means the system will serve more mobile users at a moment.

\section{CONClusion}

In this paper, we present an adaptive algorithm for dynamic joint source-channel code rate for voice traffic over LTE network. The proposed algorithm permits choosing a suboptimal solution for minimizing the number of redundant bits generated by channel coding. The output of the algorithm is a value pair of source code rate and channel code rate resulting in minimizing the number of redundant bits generated by channel coding with an acceptable MOS reduction based on some constraints on the maximum allowed end-to-end delay, maximum permitted packet loss and minimum required bandwidth. The simulation results show that in the case of the fixed "raw" packet loss, AMR-WB codec mode 2 offers the suboptimal solution with channel code rate equal to $3 / 5$. Otherwise, when the varied "raw" packet loss, AMR-WB codec mode 7 offers the suboptimal solution with channel code rate equal to $3 / 5$. The simulation results also demonstrate that the proposed algorithm always finds out the suboptimal solution which meets some constraints on given QoS information for the tradeoff between speech quality and the number of redundant bits generated by channel coding. This means there is a slightly reduction of $\operatorname{MOS}(\leq 1 \%)$ when compared to the best MOS while we can obtain the percent of decreased redundant bits generated by channel coding up to $50 \%$. This will lead to saving the bandwidth and so that the system can server more mobile users at the same time. In this study, we assume the random loss model for calculating packet loss. For the future study work, we will consider packet losses are
For the future study work, we will consider packet losses are bursty.

\section{REFERENCES}

[1] Jonghwan Hyun, Jian Li, ChaeTae Im, Jae-Hyoung Yoo, and James WonKi Hong. A volte traffic classification method in lte network. In Network Operations and Management Symposium (APNOMS), 2014 16th AsiaPacific, pages 1-6. IEEE, 2014.

[2] 3GPP. Policy and charging control architecture. TS 23.203, 3rd Generation Partnership Project (3GPP), 062011.

[3] Johnny Matta, Christine Pépin, Khosrow Lashkari, and Ravi Jain. A source and channel rate adaptation algorithm for amr in voip using the emodel. In Proceedings of the 13th international workshop on Network and operating systems support for digital audio and video, pages 92-99. ACM, 2003.

[4] J Makinen and J Vainio. Source signal based rate adaptation for gsm amr speech codec. In Information Technology: Coding and Computing, 2004. Proceedings. ITCC 2004. International Conference on, volume 2, pages 308-313. IEEE, 2004.

[5] Yicheng Huang, Jari Korhonen, and Ye Wang. Optimization of source and channel coding for voice over ip. In Multimedia and Expo, 2005. ICME 2005. IEEE International Conference on, pages 4-pp. IEEE, 2005.

[6] Fariza Sabrina and Jean-Marc Valin. Priority based dynamic rate control for voip traffic. In Global Telecommunications Conference, 2009. GLOBECOM 2009. IEEE, pages 1-8. IEEE, 2009.

[7] Mingyu Chen and Manohar N Murthi. Optimized unequal error protection for voice over ip. In Acoustics, Speech, and Signal Processing, 2004. Proceedings.(ICASSP'04). IEEE International Conference on, volume 5, pages V-865. IEEE, 2004.

[8] A Khalifeh and Homayoun Yousefi'zadeh. Optimal rate adaptation for voip over wireless tandem links. In MILITARY COMMUNICATIONS CONFERENCE, 2010-MILCOM 2010, pages 287-292. IEEE, 2010.

[9] ITUT Rec. G. 107.1, wideband e-model. Int. Telecomm. Union, Geneva, 2011.

[10] Carsten Bormann, C Burmeister, M Degermark, et al. Robust header compression (rohc). Technical report, RFC 3095, June, 2001.

[11] ITUT Rec. G. 722.2 (2003) wideband coding of speech at around $16 \mathrm{kbit} / \mathrm{s}$ using adaptive multi-rate wideband (amr-wb). International telecommunication union, Geneve, Switzerland, 2003.

[12] 3GPP. Evolved Universal Terrestrial Radio Access (E-UTRA); Multiplexing and channel coding. TS 36.212, 3rd Generation Partnership Project (3GPP), 102010.

[13] 3GPP. Evolved Universal Terrestrial Radio Access (E-UTRA); Physical layer procedures. TS 36.213, 3rd Generation Partnership Project (3GPP), 102010.

[14] Amitabha Ghosh and Rapeepat Ratasuk. Essentials of LTE and LTE-A. Cambridge University Press, 2011.

[15] Liyun Pang and Laszlo Böszörmenyi. Adaptive jitter buffer based on quality optimization under bursty packet loss. International Journal On Advances in Telecommunications, 5(1 and 2):1-10, 2012.

[16] Cristian Olariu, Micheal O Foghlu, Philip Perry, and Liam Murphy. Voip quality monitoring in lte femtocells. In Integrated Network Management (IM), 2011 IFIP/IEEE International Symposium on, pages 501-508. IEEE, 2011.

[17] Sebastian Moller, Alexander Raake, Nobuhiko Kitawaki, Akira Takahashi, and Marcel Waltermann. Impairment factor framework for wideband speech codecs. Audio, Speech, and Language Processing, IEEE Transactions on, 14(6):1969-1976, 2006.

[18] Mansour J Karam and Fouad A Tobagi. Analysis of delay and delay jitter of voice traffic in the internet. Computer Networks, 40(6):711-726, 2002.

[19] Syed A Ahson and Mohammad Ilyas. VoIP handbook: applications, technologies, reliability, and security. CRC Press, 2008.

[20] Bernard Sklar. Digital communications, volume 2. Prentice Hall NJ, 2001.

[21] Esa Piri, Jarno Pinola, Frerk Fitzek, and Kostas Pentikousis. Rohc and aggregated voip over fixed wimax: an empirical evaluation. In Computers and Communications, 2008. ISCC 2008. IEEE Symposium on, pages 1141-1146. IEEE, 2008.

[22] Yikang Xiang, Jijun Luo, and Christian Hartmann. Inter-cell interference mitigation through flexible resource reuse in ofdma based communication networks. In European wireless, pages 1-4, 2007. 